\title{
A Family of Strings with Spin
}

\author{
J. P. Krisch ${ }^{1}$
}

Received May 15, 1995

Using an equivalence theorem, we discuss some stationary interiors for a string with spin density in a space with torsion. We show that there is a family of solutions characterized by the spin divergences and compare the solutions to string solutions in general relativistic spacetimes.

\section{INTRODUCTION}

Interior solutions for infinite cosmic strings have received recent attention. The early models [1] of cosmic strings assumed an infinitely thin string with an energy density and tension along the string. The interest in more complex string interiors is motivated by the recognition that a string is a complex collection of fields [2] and may have other stress energy components besides the energy density and tension along the axis and without exact solutions there is no way of evaluating the more simple string models. Simple strings are also singular along the axis and non singular exact solutions can be very useful in discussing possible vacuum matches at the string boundary. There are three general types of single cosmic string interiors found in the literature. In general-relativistic spacetimes there are static interiors with a perfect fiuid content [3-6] and stationary interiors supporting a perfect fluid with heat flow [7]. In Einstein-Cartan spaces (with torsion) there are stationary solutions [8] containing a perfect fluid with spin density, a spin fluid. In this note we discuss some new string

1 Department of Physics, The University of Michigan, Ann Arbor, Michigan 48109, USA 
interiors with spin. We show that the solution with torsion described by Soleng [8] is the simplest member of a family of solutions. The members of the family are characterized by their divergence. We obtain the general relativistic spinning string solution with the same geometry as the new solution with torsion and compare it to the general relativistic string with heat flow discussed by Jensen and Soleng [7].

\section{STRING INTERIORS}

\subsection{The general metric and field equations}

The metric we consider is

$$
d s^{2}=-F(d t+k d \phi)^{2}+d r^{2}+d z^{2}+D^{2} d \phi^{2} .
$$

Jensen and Soleng [7] discuss one stationary solution where the string interior is accelerated but most interiors assume an unaccelerated string interior. We shall assume

$$
F=1 \text {. }
$$

The general relativistic field equations for a string with a perfect spin fluid stress energy content in a space without torsion are

$$
\begin{aligned}
(00) \quad \epsilon & =\frac{3}{4}\left(\frac{k^{\prime}}{D}\right)^{2}-\frac{k^{\prime} S_{r \phi}}{D^{2}}-\frac{D^{\prime \prime}}{D}, \\
(z z) \quad p_{3} & =-\frac{1}{4}\left(\frac{k^{\prime}}{D}\right)^{2}+\frac{D^{\prime \prime}}{D}, \\
(r r)(\phi \phi) \quad p_{1}=p_{2} & =\frac{1}{4}\left(\frac{k^{\prime}}{D}\right)^{2}-\frac{k^{\prime} S_{r \phi}}{D^{2}}, \\
(0 \phi)\left(\frac{k^{\prime}-S_{r \phi}}{D}\right)^{\prime} & =0 .
\end{aligned}
$$

Derivatives are with respect to the $r$ coordinate and denoted by prime. These field equations follow from the spin fluid stress energy tensor developed by Ray and Smalley [9] which is applicable to both spaces with and without torsion.

The static and stationary general relativistic solutions for the spacetime of eq. (1) can be discussed using these equations. We will not write down the Einstein-Cartan field equations for spacetimes with torsion as those solutions will be obtainable from the general relativistic static solution by an equivalence theorem [10]. This will be shown below. 
The field equations are tetrad indexed using the tetrads that diagonalize the metric. These tetrads are chosen so that the time like component lies along the comoving velocity, $u^{i}$ :

$$
\begin{aligned}
& e_{(0)}^{i}=(1,0,0,0) \\
& e_{(r)}^{i}=(0,1,0,0) \\
& e_{(z)}^{i}=(0,0,1,0) \\
& e_{(\phi)}^{i}=\left(\frac{-k}{D}, 0,0, \frac{1}{D}\right) .
\end{aligned}
$$

Tetrad indices are in parentheses and spacetime indices are not. The spin content of the field equations is discussed in the next section.

\subsection{Spin content}

The field equations given in eqs. (3)-(6) are derived using the spin fluid Lagrangian discussed by Ray and Smalley [9]. The spin density in the fluid, $S_{i j}$, is defined as

$$
S_{i j}=\rho \sigma\left(e_{i}^{(r)} e_{j}^{(\phi)}-e_{j}^{(r)} e_{i}^{(\phi)}\right)
$$

where $\sigma$ is a spin module function and $\rho$ the proper density of the fluid. The spin density is related to the vector spin density by

$$
S^{i}=\frac{e^{i j k l} u_{j} S_{k l}}{2 \sqrt{-g}}
$$

Limiting the spin density to $S_{r \phi}$ is equivalent to assuming that the spin is oriented along the z-axis.

The divergence of the spin density is

$$
W^{i}=\left(S^{i j}\right)_{; j} .
$$

The divergence has a different form depending on whether or not the connections of the spacetime include torsion. For a general relativistic spacetime without torsion the two non zero tetrad indexed components of the divergence are.

$$
\begin{aligned}
& W^{(0)}=k^{\prime} S^{r \phi} \\
& W^{(\phi)}=\frac{d}{d r}\left(D S^{\phi r}\right)=\frac{d}{d r}\left(-S^{z}\right) .
\end{aligned}
$$


For an Einstein-Cartan spacetime with torsion the diverence components are

$$
\begin{aligned}
W^{(0)} & =\left(k^{\prime}-S_{r \phi}\right) S^{r \phi} \\
W^{(\phi)} & =\frac{d}{d r}\left(D S^{\phi r}\right)=\frac{d}{d r}\left(-S^{z}\right)
\end{aligned}
$$

We will see that the divergence of the spin density can be used to characterize the solutions discussed in this paper. It also allows insights into the spin structure. The timelike component of the divergence can be calculated directly or it can be simply obtained in a general form from the Frenkel condition on the spin

$$
u_{i} S^{i j}=0
$$

Taking the derivative with respect to $j$ and using the standard expansion of the velocity covariant derivative this equation may be written as

$$
W^{(0)}=S^{i j} \omega_{i j(\mathrm{RC})}=S^{i j}\left(\omega_{i j}-\frac{S_{i j}}{2}\right),
$$

where $\omega_{i j(\mathrm{RC})}$ is the vorticity in the torsional spacetime and $\omega_{i j}$ is the usual GR fluid vorticity. The equivalence enforces a zero timelike divergence component. This is a statement that in this stationary fluid, the usual GR fluid vorticity is directed oppositely to the spin. A very rough analogy that is helpful in visualization can be made between the spin fluid and some atomic states with $j=O, \ell, s \neq 0$. The spacelike divergence component, from eq. (12) or (14), will determine the functional form of the spin in a modeling calculation. It also carries information about the vector nature of the spin since this component is proportional to the curl of the vector spin density, $S^{i}$.

\subsection{String interior solutions}

For each of the solutions, the functions to be determined are $D, k$ and the pressures, energy density and spin density.

\subsubsection{The static string}

The perfect fluid static string has been discussed by Hiscock [3], Linet $[4,6]$ and Gott [5]. The stress energy content is $\epsilon=\lambda, p_{2}=-\lambda, p_{r}=p_{\phi}=0$, $k=0, S_{r \phi}=0$. The field equations give the interior geometry

$$
\left(d s_{1}\right)^{2}=-d t^{2}+d r^{2}+d z^{2}+D^{2} d \phi^{2}
$$


and with the assumption that the metric will be flat on the axis one has

$$
D=\frac{\sin (\sqrt{\lambda} r)}{\sqrt{\lambda}}
$$

2.3.2. The Einstein-Cartan stationary string with spin-solution from equivalence

The stationary Einstein-Cartan string has been discussed by Soleng [8]. The stress energy content is $\epsilon=\lambda, p_{2}=-\lambda, p_{r}=p_{\phi}=0, k=0$, $S_{r \phi}=S_{0} D$. The geometry is

$$
d s^{2}=-(d t+k d \phi)^{2}+d r^{2}+d z^{2}+D^{2} d \phi^{2}
$$

with

$$
D=\frac{\sin (\sqrt{\lambda} r)}{\sqrt{\lambda}} \quad \text { and } \quad k=\frac{S_{0}}{\lambda}(1-\cos (\sqrt{\lambda} r)) .
$$

The stationary string in the spacetime with torsion follows directly from the static string in general relativity using the equivalence shown by Krisch and Smalley [10]. The equivalence was originally stated for isotropic pressures but is also true for anisotropic fluids. For static metrics with only radial dependence, the statement of equivalence is as follows. Every static perfect fluid solution $\left(g_{i j}, \epsilon, p_{i}, D\right)$ in general relativity is equivalent to a stationary Einstein-Cartan solution $\left(g_{i j}^{*}, \epsilon, p_{i}, D, S_{i j}\right)$ with

$$
\begin{aligned}
g_{i j} & =g_{i j}^{*}, \quad i, j=r, z, t \\
g_{0 \phi} & =-k \quad \text { with } \quad\left(g_{00} k^{\prime}-k g_{00}^{\prime}\right) g_{00}^{\prime}=0 \\
S_{r \phi} & =\frac{\left(-g_{00} k^{\prime}+k g_{00}^{\prime}\right)}{\sqrt{-g_{00}^{3}}} .
\end{aligned}
$$

For the unaccelerated string solutions considered here these simplify to

$$
\begin{aligned}
g_{i j} & =g_{i j}^{*}, \quad i, j=r, z, t \\
g_{0 \phi}^{*} & =-k, \\
S_{r \phi} & =k^{\prime} .
\end{aligned}
$$

Using the relation between spin density and spin, the last equation can be written as

$$
k^{\prime}=D S^{z}
$$


If the spin density is constant

$$
S^{z}=S_{0}
$$

and using the static $D$ function required by the equivalence we obtain

$$
k=\frac{S_{0}}{\lambda}(1-\cos (\sqrt{\lambda} r)) \text {. }
$$

This interior is the solution found by Soleng [8] for a spinning string in an Einstein-Cartan spacetime. The equivalence makes it clear that this is only one of a family of spinning string solutions and other members of the family can be generated from eq. (20). The assumption in eq. (21) that the spin is constant is one of many possible choices. The equivalence produces a spin in the Einstein-Cartan spacetime with a zero timelike divergence component for any functional choice of spin. The Soleng constant spin choice [8] corresponds to a spin density with a zero spacelike component also. Other choices for the spins would lead to more complex vorticity and divergence behavior but would not change the fluid's energy density or pressures. The energy and pressure remain the static general relativistic values.

\subsubsection{Another solution belonging to the equivalence family}

Soleng's solution [8] has a zero divergence and the next family member one could consider would be one with a constant divergence $W^{(\phi)}=W_{0}$,

$$
S^{z}=W_{0}(R-r)
$$

with $R$ a constant. The associated tensor density is

$$
S_{r \phi}=D W_{0}(R-r) \text {. }
$$

Using the static $D$ function that is appropriate for this family we have

$$
k^{\prime}=W_{0}(R-r) \frac{\sin (\sqrt{\lambda} r)}{\sqrt{\lambda}} .
$$

Integrating eq. (24) one obtains

$$
k=\frac{-W_{0}}{\sqrt{\lambda}}\left\{(R-r) \cos [\sqrt{\lambda} r]-\left(\frac{\sin (\sqrt{\lambda} r)}{\sqrt{\lambda}}\right)+k_{1}\right\}
$$

$k_{1}$ is a constant of integration

This solution describes a string with the same energy and pressure as the static string but with a spin density and vorticity that vanish at the point $r=R$. For comparison it is of interest to find the fluid parameters for the general relativistic solution that has this same geometry. 


\section{THE GENERAL RELATIVISTIC STATIONARY STRING WITH SPIN}

\subsection{The solution}

We start by solving eq. (6). The solution is

$$
k^{\prime}=S_{r \phi}+k_{2} D
$$

We assume $k_{2}=O$ in order to examine the same kind of solution as in the Einstein-Cartan interior and assume that $S_{r \phi}$ is given by eq. (23). Using the static $D$ function, one obtains the same function for $k$, eq. (25). The metric for this solution is them formally identical to the metric for the constant divergence Einstein-Cartan solution discussed in Section 3. The fluid parameters follow from the field equations

$$
\begin{aligned}
\varepsilon & =\lambda-\frac{W_{0}^{2}(R-r)^{2}}{4} \\
p_{z} & =-\lambda+\frac{W_{0}^{2}(R-r)^{2}}{4} \\
p_{r}=p_{\phi} & =\frac{-3 W_{0}^{2}(R-r)^{2}}{4} \\
S_{r \phi} & =D W_{0}(R-r)
\end{aligned}
$$

or

$$
S^{z}=W_{0}(R-r)
$$

For this interior, the spin density tensor $S_{r \phi}$ approaches zero on the axis but in such a way that the spin vector density $S^{z}$ is a constant. The spin divergences follow from eqs. (11) and (12).

$$
\begin{aligned}
& W^{(0)}=W_{0}^{2}(R-r)^{2} \\
& W^{(\phi)}=W_{0} .
\end{aligned}
$$

Comparing this solution to the Einstein-Cartan constant divergence solution with the same geometry, we see that the general relativistic string with spin has a much more complex fluid behavior. One might think that a more complex spin behavior in the Einstein-Cartan interior would generate a more complex stress-energy content, but it does not. Changing the spin in the family of Einstein-Cartan solutions described above modifies the fluid vorticity and the spin divergence. A very interesting insight 
follows from comparing this general relativistic solution to the solution of for a string with heat flow [7]. The field equations considered by Jensen and Soleng are equations (3)-(5) with $S_{r \phi}=0$. They modified eq. (6) in order to generate interesting solutions by adding heat flow to their fluid. Equation (6) becomes

$$
k^{\prime}=2 D \alpha \lambda(R-r) \text {. }
$$

A static $D$ function is used. A comparison of eq. (30) to eq. (24) shows that the geometry of the spinning string in general relativity and the general relativistic string with heat flow are formally the same with $2 \alpha \lambda$ replacing $W_{0}$. The fluid parameters are similar but a different assumption about the form of the energy leads to slightly different coefficients.

\section{CONCLUSIONS}

We have shows that the constant spin string interior in a spacetime with torsion is only one of a family of string interiors characterized by the spin divergence. We present a second Einstein-Cartan interior which has the same geometry as either the general relativistic spinning string or the general relativistic spin with heat flow. The energy and pressures for the string with torsion are the same as those for the static string. The general relativistic solutions have a more complex fluid behavior. Since a string interior is a field or set of fields, the motivation for choosing an interior with spin over one with heat flow depends on the physics of the problem being considered. Choosing a spacetime with torsion over one without torsion is a question that will be answered experimentally.

\section{REFERENCES}

1. Vilenkin, A. (1981). Phys. Rev. D23, 852.

2. Garfinkle, D. (1985). Phys. Rev. D32, 1323.

3. Hiscock, W. A. (1985). Phys. Rev. 31, (Brief Report) 3288.

4. Linet, B. (1985). Gen. Rel. Grav. 17, 1109.

5. Gott, J. R., III (1985). Astrohpys. J. 288, 422.

6. Linet, B. (1986). J. Math. Phys. 27, 1817.

7. Jensen, B., and Soleng, H. H. (1992). Phys. Rev. D45, 3528.

8. Soleng, H. H. (1992). Gen. Rel. Grav. 24, 111; (1994). Phys. Rev. D49, 1124.

9. Ray, J. R., and Smalley, L. L. (1982). Phys. Rev. D26, 2619; (1983). Phys. Rev. Lett. 49, 1059; (1983). Phys. Rev. Lett. 50, 626E; (1983). Phys. Rev. D27, 1382.

10. Krisch, J. P., and Smalley, L. L. (1993). Class. Quant. Grav. 10, 995. 